\title{
Mean-Field Approach to Dielectric Relaxation in Giant Dielectric Constant Perovskite Ceramics
}

\author{
Shanming Ke, ${ }^{1,2}$ Peng Lin, ${ }^{1,2}$ Haitao Huang, ${ }^{3}$ Huiqing Fan, ${ }^{4}$ and Xierong Zeng ${ }^{1,2}$ \\ ${ }^{1}$ College of Materials Science and Engineering, Shenzhen University, Shenzhen 518060, China \\ ${ }^{2}$ Shenzhen Key Laboratory of Special Functional Materials, Shenzhen 518060, China \\ ${ }^{3}$ Department of Applied Physics and Materials Research Center, The Hong Kong Polytechnic University, Hong Kong \\ ${ }^{4}$ School of Materials Science and Engineering, Northwestern Polytechnical University, Xian 710072, China \\ Correspondence should be addressed to Shanming Ke; smke@szu.edu.cn and Haitao Huang; aphhuang@polyu.edu.hk
}

Received 25 June 2013; Accepted 25 August 2013

Academic Editor: Matjaz Valant

Copyright (c) 2013 Shanming Ke et al. This is an open access article distributed under the Creative Commons Attribution License, which permits unrestricted use, distribution, and reproduction in any medium, provided the original work is properly cited.

\begin{abstract}
The dielectric properties of $\mathrm{CaCu}_{3} \mathrm{Ti}_{4} \mathrm{O}_{3}(\mathrm{CCTO})$ and $\mathrm{A}_{2} \mathrm{FeNbO}_{6}(\mathrm{AFN}, \mathrm{A}=\mathrm{Ba}$, $\mathrm{Sr}$, and $\mathrm{Ca}$ ) giant dielectric constant ceramics were investigated in the frequency range from $1 \mathrm{~Hz}$ to $10 \mathrm{MHz}$. The relaxation properties can be perfectly described by a polaron model, indicating that the dielectric relaxation is intimately related to the hopping motion caused by localized charge carriers.
\end{abstract}

\section{Introduction}

As driven by the impetus of smaller and smaller feature size of devices in microelectronics, researchers are looking for the so-called high- $k$ materials. Perovskites such as lead zirconate titanate (PZT) usually possess high dielectric constant of a few hundreds at room temperature $[1,2]$ and are widely used as capacitive components. The perovskite-based oxide $\mathrm{CaCu}_{3} \mathrm{Ti}_{4} \mathrm{O}_{12}$ (CCTO, space group Im3) was reported to have a colossal dielectric constant (CDC) in the order of $10^{4}$ which is nearly constant from $400 \mathrm{~K}$ down to $100 \mathrm{~K}$ but drops rapidly to less than 100 below $100 \mathrm{~K}$ [3]. A huge amount of work [3-7] has thereafter been carried out in attempts to understand the origin of the remarkable dielectric properties. It has been found that the temperature at which the steplike decrease in dielectric constant takes place strongly depends on the measuring frequency and roughly follows an Arrhenius behavior. Similar phenomenon has also been reported in a number of materials, such as $\mathrm{A}_{2} \mathrm{FeBO}_{6}(\mathrm{~A}=$ $\mathrm{Ba}, \mathrm{Sr}$, and $\mathrm{Ca} ; \mathrm{B}=\mathrm{Nb}, \mathrm{Ta}$, etc.) [8], $\mathrm{La}_{1-x} \mathrm{Ca}_{x} \mathrm{MnO}_{3}$ (LCMO) [9], $\mathrm{Pr}_{0.7} \mathrm{Ca}_{0.3} \mathrm{MnO}_{3}$ (PCMO) [10], $\mathrm{LaCuLiO}_{4}$ [11], $\mathrm{LaSrNiO}_{4}$ [12], $\mathrm{TbMnO}_{3}$ [13], and $\mathrm{Li} / \mathrm{Ti}$ doped $\mathrm{NiO}$ [14]. The remarkable low temperature dielectric relaxation in many manganites, cuprates, and nickelates $[9-12,15-17]$ has been attributed to localized hopping of polarons between lattice sites with a characteristic timescale. Furthermore, this relaxation behavior clearly displays a freezing temperature following a glass-like process, suggesting that an electronic glass state is realized. It seems plausible to think of the influence of the electric state on the dielectric response of a solid.

Polaronic relaxation usually involves a variable range hopping (VRH) or a nearest-neighbor hopping conduction process. Polarons as the main sources contributing to the conduction of CCTO ceramics at the low temperature relaxation region has been confirmed [18]. Tselév et al. [19] found a power-law frequency dependent dielectric response in CCTO epitaxial thin films suggesting that the dielectric contribution came from hopping carriers. Based on these results, the low temperature dielectric response in CCTO has been attributed to polaron relaxation related localized charge carriers [20]. It should be mentioned that Maxwell-Wagner (MW) origin, such as the internal barrier layer capacitance (IBLC) should not be excluded in the explanation of the colossal dielectric constants, especially at high temperatures $[4,6,7,21]$. Although, the MW type mechanisms which were resulted from the surface layer [21], IBLC [4], and/or contact electrode [6] do have an influence on the dielectric constant 
values of CDC materials, they nearly have no influence on the relaxation process at low temperature.

In this paper, we present a systematic study on the low temperature dielectric relaxation process of CCTO and $\mathrm{A}_{2} \mathrm{FeNbO}_{6}(\mathrm{AFN}, \mathrm{A}=\mathrm{Ba}, \mathrm{Sr}$, and $\mathrm{Ca}$ ) systems. A polaron relaxation model was proposed as a possible explanation to the low temperature dielectric relaxation in CCTO and AFN ceramics. It should be mentioned that MW model and polaron model are both related to the moving of space charge carriers. The difference is that MW model emphasizes the interface, but polaron model considers the space electrons/ions exist in the whole bulk materials. Although the polaron concept has been used to explain the dipolar effects induced by charge carrier hopping motions inside the CCTO grains $[20,22]$, a clear theory formula to describe polaron relaxation is absence. Based on polaron theory, Jonscher's law (universal dielectric response (UDR)) could be used to fit the frequency dependence of the dielectric permittivity. By using a mean-field approach, we will present a simplicity expression on the temperature dependence of the dielectric permittivity driven from polaron relaxation.

\section{Experimental}

Single phase CCTO and AFN (A = Ba, Sr, and $\mathrm{Ca}$ ) ceramics were prepared through a conventional mixed oxide route, and the detailed processing parameters can be found elsewhere $[23,24]$. The Wolframite method, using $\mathrm{FeNbO}_{4}$ as the $B$ site precursor, has been used to synthesize AFN with no secondary iron-oxide phases.

$\mathrm{X}$-ray diffraction (XRD) was conducted on sintered ceramics samples of CCTO and AFN. Data were collected on an automated diffractometer (X'Pert PRO MPD, Philips) with $\mathrm{Cu} K_{\alpha 1}$ radiation. The XRD results confirm that the sintered ceramics are single phase. The fracture surfaces of the ceramic pellets were examined by scanning electron microscopy (SEM, JSM-6335F, and JEOL). Micro-Raman spectral measurements were performed on a JY HR800 Raman spectrometer under backscattering geometry. An Argon ion laser was used as the excitation source with an output power of $15 \mathrm{mw}$ at $488 \mathrm{~nm}$. For dielectric measurement, top and bottom electrodes were made by coating silver paint on both sides of the sintered disks and followed by a firing at $650^{\circ} \mathrm{C}$ for 20 minutes. The temperature and the frequency dependences of the dielectric permittivity of the samples were measured by a frequency response analyzer (Novocontrol Alpha-analyzer) over a broad frequency range (1 Hz-10 MHz).

\section{Results and Discussion}

3.1. Crystal Structure and Microstructure. Figure 1 shows the room temperature XRD pattern of sintered CCTO ceramics. All the diffraction peaks can be indexed according to a cubic cell of space group $\operatorname{Im} 3$ [3]. Standard group theory analysis predicts that the Raman active modes are distributed among the irreducible representations as $2 A_{g}+2 E_{g}+4 F_{g}$ [25]. Inset of Figure 1 shows the Raman spectrum at room

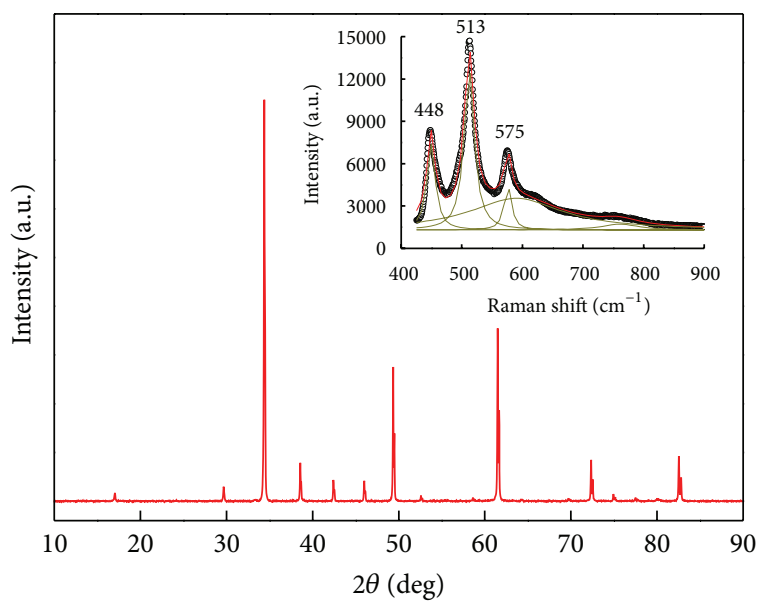

FIGURE 1: XRD pattern of CCTO ceramics. The inset shows the micro-Raman scattering spectra.

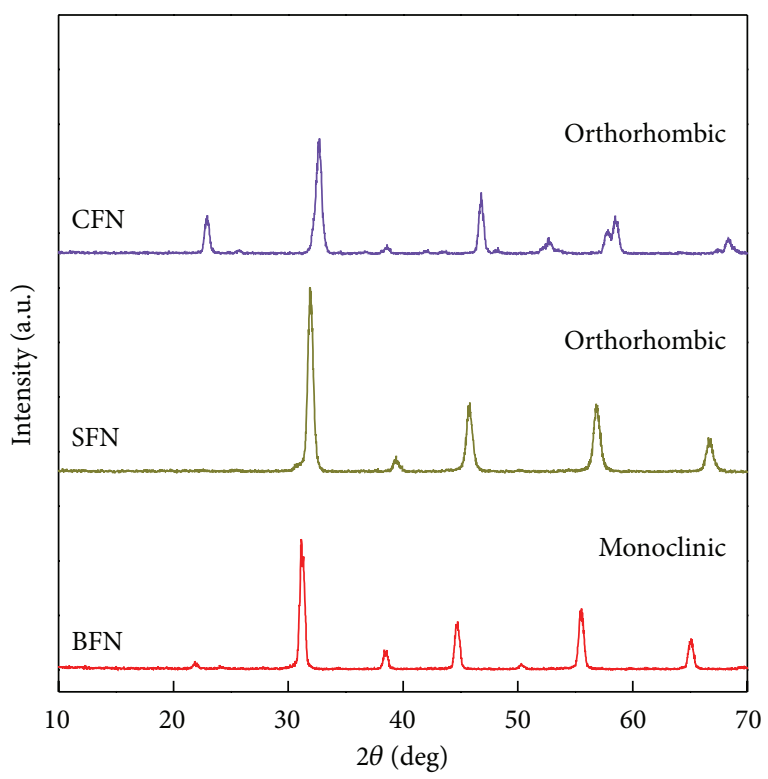

FIGURE 2: XRD patterns of BFN, SFN, and CFN ceramics.

temperature. The Raman lines at 448 and $512 \mathrm{~cm}^{-1}$ have exact $A_{g}$ symmetry, and a line of $F_{g}$ symmetry is clearly pronounced at $575 \mathrm{~cm}^{-1}$, which is assigned to the Ti-O$\mathrm{Ti}$ antistretching mode of the oxygen octahedra [25]. The Raman spectrum also confirms a single cubic phase of our CCTO sample.

The XRD patterns of the $\mathrm{Ba}_{2} \mathrm{FeNbO}_{6}(\mathrm{BFN}), \mathrm{Sr}_{2} \mathrm{FeNbO}_{6}$ (SFN), and $\mathrm{Ca}_{2} \mathrm{FeNbO}_{6}(\mathrm{CFN})$ are given in Figure 2. The diffraction peaks of BFN samples can be indexed according to a monoclinic structure [26], while SFN and CFN samples show an orthorhombic structure with the space group Pnma(62) [27, 28]. Figure 3 displays typical SEM photographs of the fracture surface of CCTO and SFN ceramics. The average grain size of CCTO is found to be in the range of $2 \sim 8 \mu \mathrm{m}$, while SFN exhibits a morphology with the bimodal distribution of grain size. One has the grain size of several 


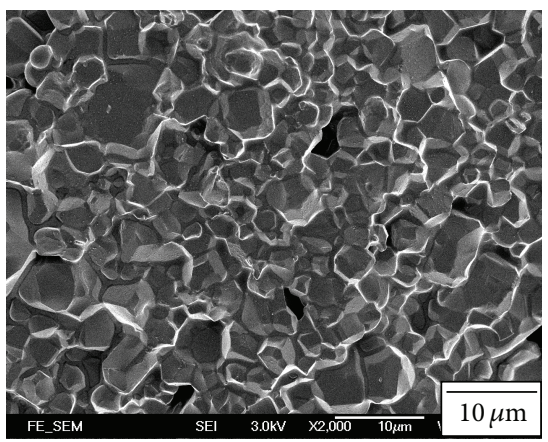

(a)

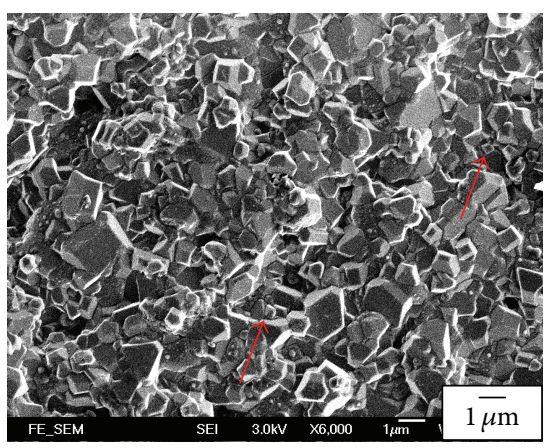

(b)

Figure 3: SEM images of the fracture surface of (a) CCTO and (b) SFN ceramics. The arrows denote nanograins in SFN.

micrometers, and the other has the smaller grain size in the order of several hundreds of nanometers. It should be noted that $\mathrm{BFN}$ and $\mathrm{Ca}_{2} \mathrm{FeNbO}_{6}$ (CFN) display a similar morphology to that of SFN, while BFN has a slightly larger average grain size than SFN and CFN.

3.2. Dielectric Behavior. Figure 4 shows the frequency dependences of the real $\left[\varepsilon^{\prime}(f)\right]$ and imaginary $\left[\varepsilon^{\prime \prime}(f)\right]$ parts of the dielectric constant of CCTO, BFN, CFN, and SFN under different temperatures. The temperatures were chosen because they reveal the typical relaxation features of the mentioned ceramics. The high frequency region of CCTO and BFN shows the presence of a Debye-like dipolar relaxation process, and SFN and CFN show a generalized dipolar loss peak possessing a more serious departure from Debye, as summarized by Jonscher [29]. The peak frequency of $\varepsilon^{\prime \prime}(f)$ decreases with decreasing temperatures for all the samples, indicating the freezing in dipolar moment. The width of the $\varepsilon^{\prime \prime}(f)$ peak of AFN (A = Ba, Sr, Ca) is much larger than that of CCTO (as shown in Figure 4), which implies a broader distribution of relaxation time. Above the $\varepsilon^{\prime \prime}(f)$ peak frequencies, the high-frequency branches of CCTO and AFN follow the fractional power law [29]:

$$
\varepsilon^{\prime \prime}(\omega)=\cot \left(\frac{n \pi}{2}\right) \varepsilon^{\prime}(\omega) \propto(i \omega)^{n-1},
$$

where $\omega$ is the angular frequency $(2 \pi f)$ and $n$ is the exponential constant. The calculated exponent $n$ from Figure 4 is $0.11,0.12,0.25$, and 0.38 for CCTO, BFN, CFN, and SFN, respectively. It should be noted that $n$ is nearly independent of temperature for all these CCTO and AFN samples. Larger $n$ means a much lower loss $\left(\varepsilon^{\prime \prime}(\omega) / \varepsilon^{\prime}(\omega)=\cot (n \pi / 2)\right)$ and flatter frequency dependence than that in a Debye relaxation.

The peak frequency of the above relaxation process obeys the following Arrhenius law:

$$
f=f_{0} \exp \left(-\frac{E_{a}}{k T}\right),
$$

where $f_{0}$ is a prefactor and $E_{a}$ is the activation energy. The Arrhenius fits for CCTO, BFN, SFN, and CFN ceramics are summarized in Figure 5, where $E_{a}=0.1,0.18,0.23$, and $0.34 \mathrm{eV}$ for CCTO, BFN, SFN, and CFN, respectively. The obtained values are very close to the previous reported ones for the same materials $[4-7,30]$.

Although, the Maxwell-Wagner type mechanism is always responsible for the dielectric permittivity of ceramics as an extrinsic source due to the existence of grain boundaries. This model cannot explain why the one with very similar microstructure and electrical resistivity (BFN, CFN, and SFN) shows so different relaxation regions and activation energies. In addition, in terms of the Maxwell-Wagner model, it is very difficult to explain the similar dielectric relaxation behavior appearing in the same temperature region for single crystals, polycrystalline ceramics, and epitaxial thin films whose microstructure is certainly different. In Figure 6, the imaginary part of dielectric permittivity $\varepsilon^{\prime \prime}$ of CCTO and BFN is shown as a function of the real part $\varepsilon^{\prime}$, namely, the Cole-Cole plot. From Figure 6, all the data at different temperatures collapse on an arc described by the Cole-Cole equation, showing universal scaling behavior in the dielectric response. We note that in the lower frequency region there is a relaxation relating to the extrinsic effect. This relaxation is probably due to a Schottky-type barrier existing in this ceramic [31].

3.3. Mean-Field Approach. In a previous study, It has been confirmed that localized charge carriers are the main sources contributing to the conduction of CCTO [18] and AFN [32] ceramics at low temperature relaxation region. Under an external electric field, the hopping motion of localized carriers like polarons gives rise to dipolar effect and sizable polarization contribution to the dielectric permittivity. Therefore a quantitative description of the polaron contribution to the dielectric response is highly demanding.

Ramirez et al. [33] have proposed a defect model as a possible explanation of the low temperature dielectric response of CCTO. In this model, isolated defects such as $\mathrm{Cu}$ vacancies produce a local disruption of the ideal cubic structure and then the defective regions relax between alternate equivalent configurations. However, high resolution experiments [33] have revealed that $\mathrm{Cu}$ vacancies most probably exist in the grain boundary regions, and thus they cannot be shown in the bulk response. Therefore, polarons are mainly responsible for the bulk dielectric relaxation. Essentially, it is reasonable to presume that polarons are isolated; then the cooperative effects are absent at low densities of polarons. The relaxation of polarons occurs at the rate $\gamma$ determined by the energy 


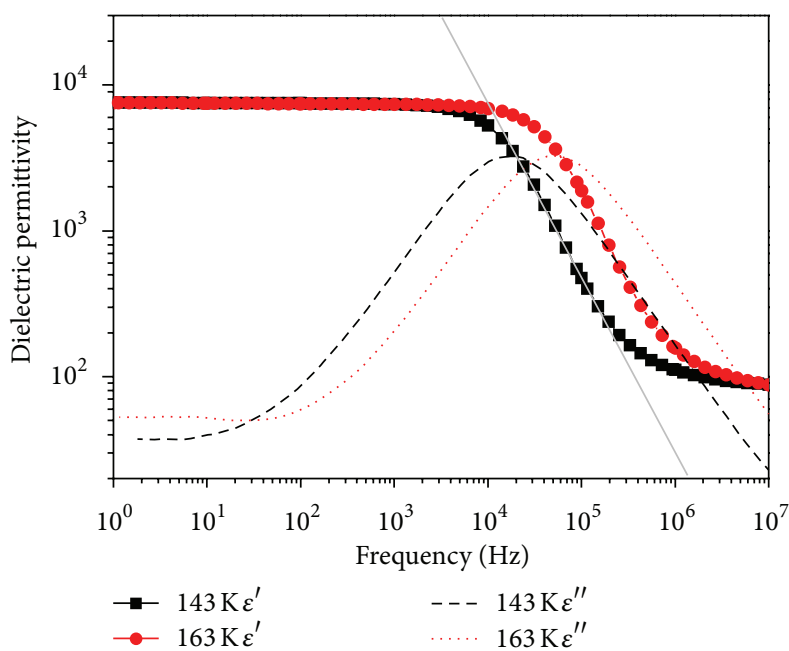

(a)

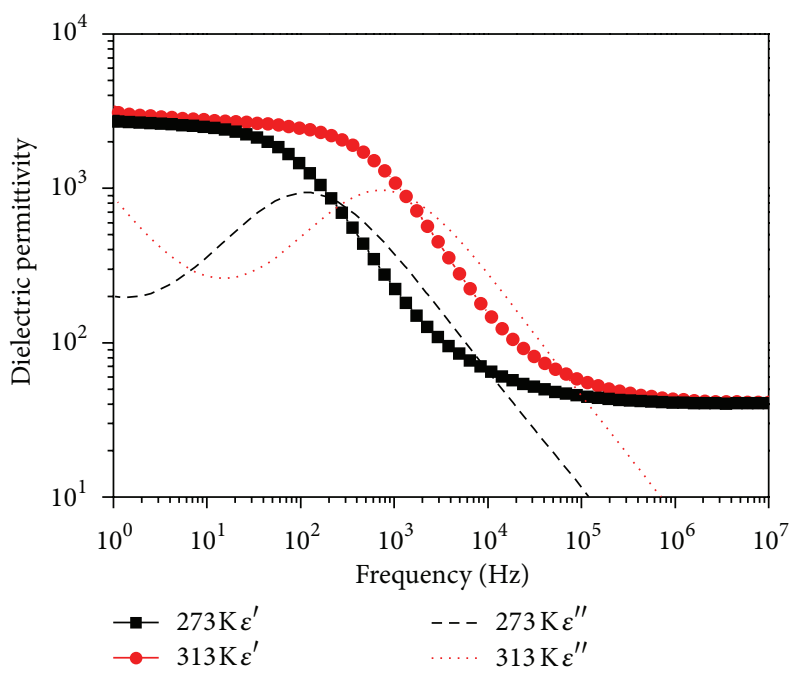

(c)

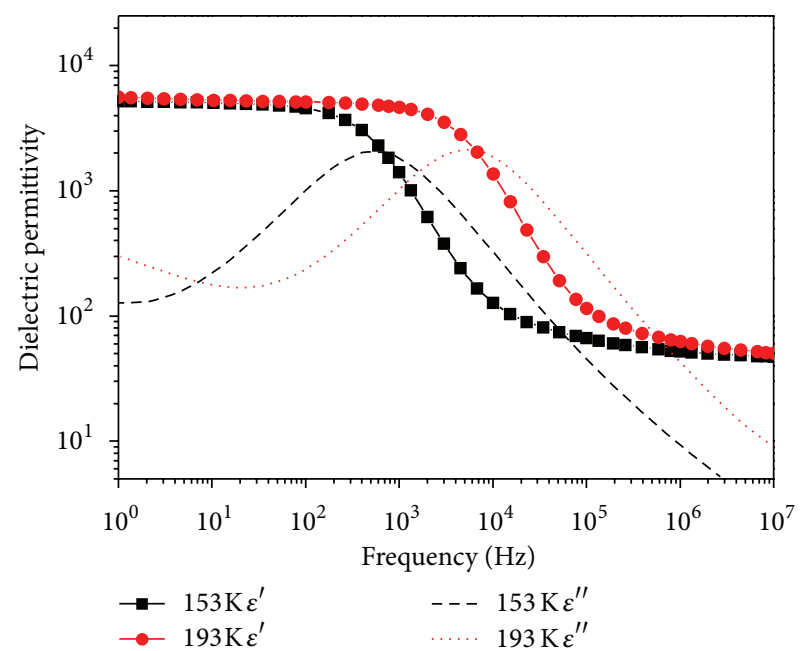

(b)

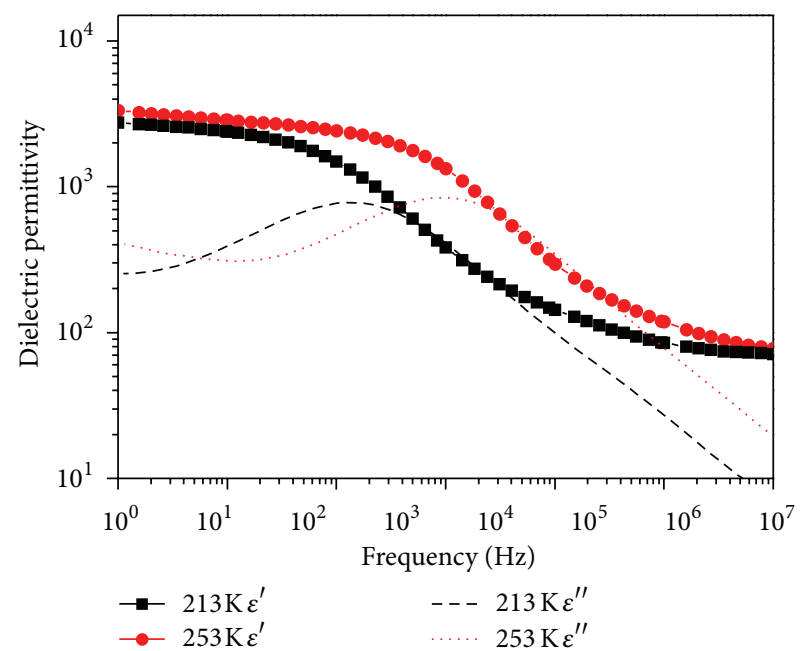

(d)

FIGURE 4: Frequency dependence of the real and imaginary parts of dielectric permittivity for (a) CCTO, (b) BFN, (c) CFN, and (d) SFN. The straight gray line in (a) is a guide to the eye.

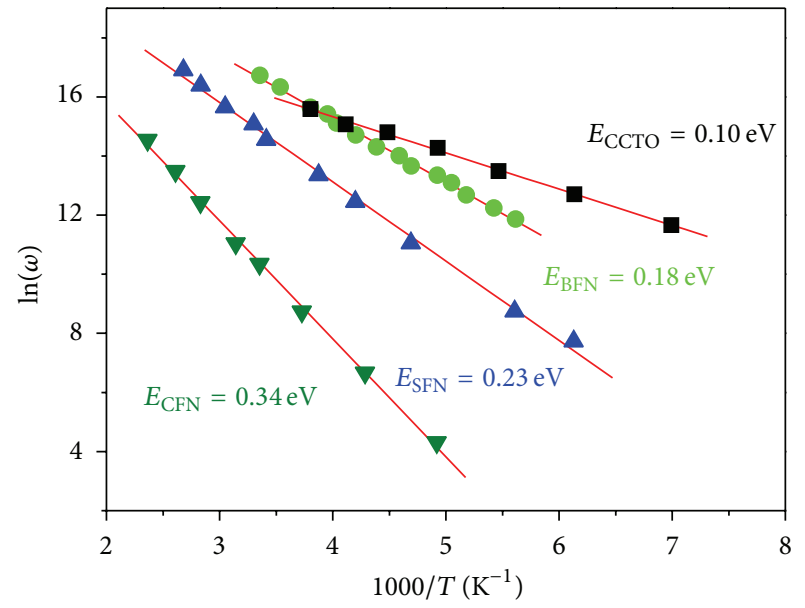

FIGURE 5: Arrhenius fit of the frequency dependence of the dynamic transition temperatures $T_{m}$ for the dielectric relaxation in CCTO, $\mathrm{BFN}, \mathrm{SFN}$, and CFN. barrier between alternate equivalent configurations and the temperature:

$$
\gamma=\gamma_{0} \exp \left(\frac{-\Delta}{T}\right), \quad\left(\Delta=\frac{E}{k_{B}}\right)
$$

where $\gamma_{0}$ depends on the effective mass of the defect and is in the order of $10^{10}-10^{12} \mathrm{~Hz}$. The local polarizability of a polaron is

$$
\chi_{p}(\omega, T)=\frac{\mu_{p} \gamma}{-i \omega+\gamma},
$$

where $\mu_{p}$ is the static polarizability of a polaron. It should be noted that (3) and (4) could be obtained directly from [33]. As suggested by Ramirez et al. [33], a mean-field approximation 


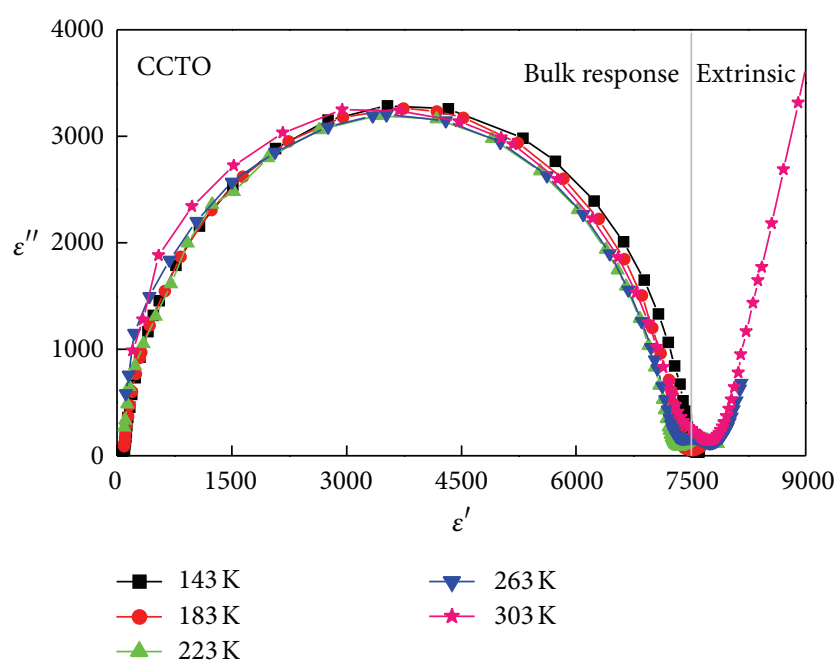

(a)

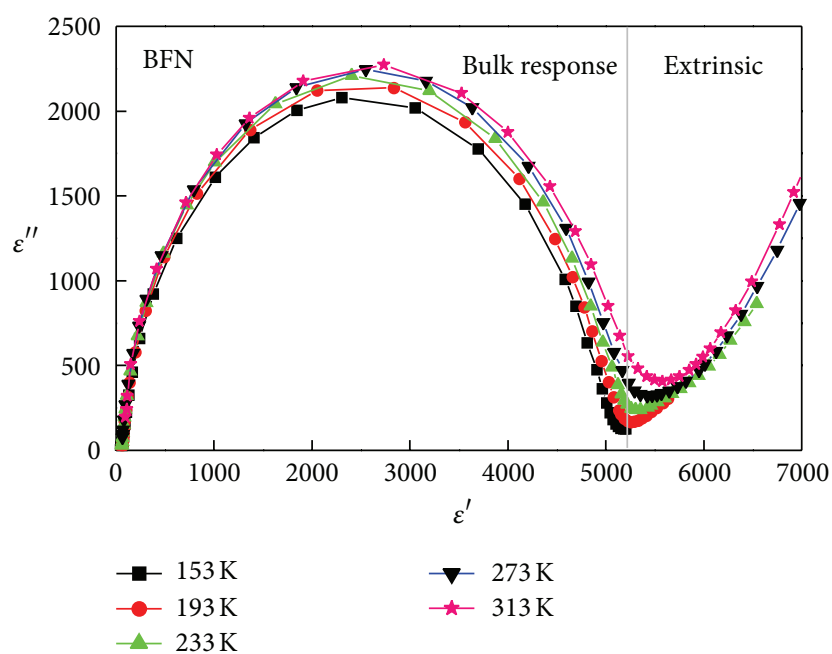

(b)

FIGURE 6: The imaginary part of dielectric permittivity $\varepsilon^{\prime \prime}$ of CCTO and $\mathrm{BFN}$ is plotted against the real part $\varepsilon^{\prime}$.

can be adopted and the dielectric response can be calculated by the Clausius-Mossotti equation:

$$
\begin{aligned}
\varepsilon(\omega, T) & \approx \frac{\varepsilon_{0}}{1-(4 \pi / 3) \rho \varepsilon_{0} \chi_{p}} \\
& =\frac{\varepsilon_{0}}{1-(4 \pi / 3) \rho \varepsilon_{0}\left(\mu_{p} \gamma /(-i \omega+\gamma)\right)},
\end{aligned}
$$

where $\rho$ is the density of the polarons, and the details can be found in [34]. It is worth noting that the Clausius-Mossotti equation works best for gases and is only approximately true for liquids or solids, particularly for polar materials, or the dielectric constant is large. In addition, the limitations of the approximation in (5), which does not consider the variations around each polaron properly, should also be kept in mind.
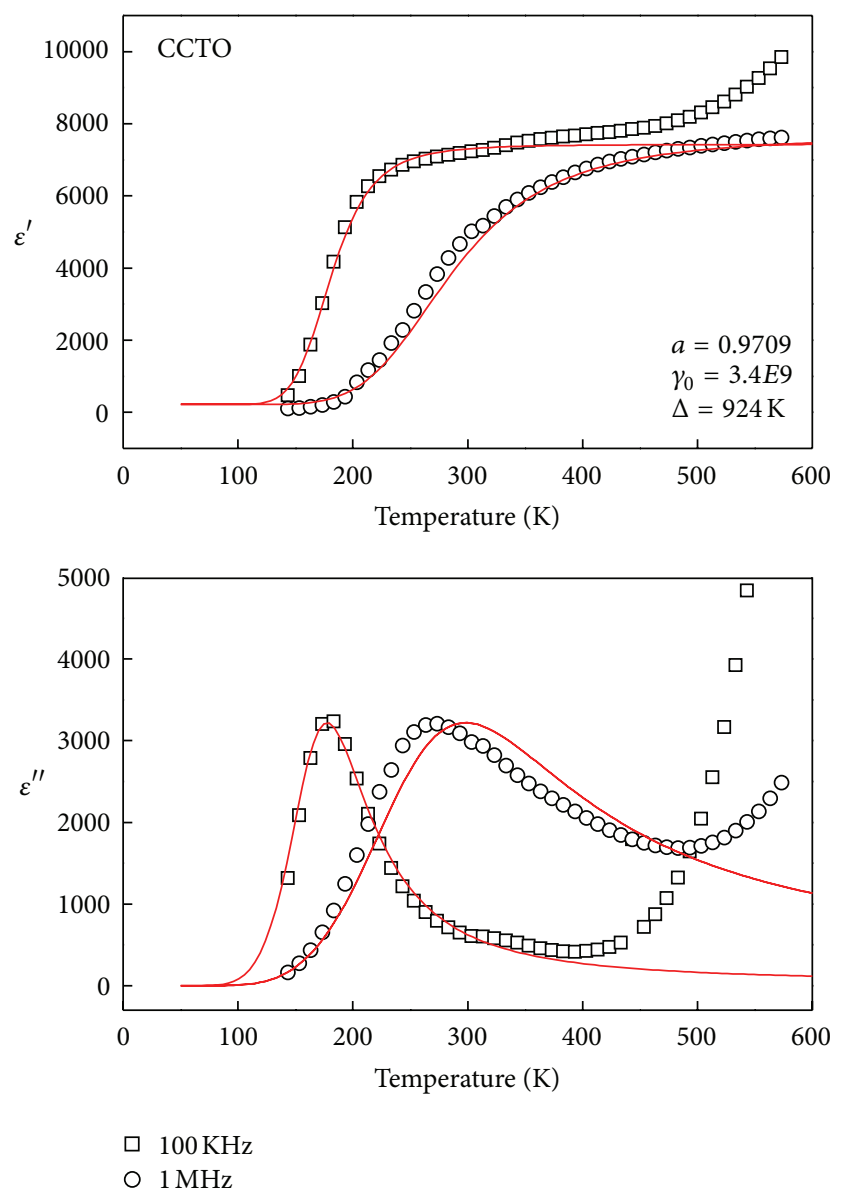

FIGURE 7: The temperature dependence of dielectric constant of CCTO. The solid lines are the best fits from polaron model (see text).

Let $a=(4 \pi / 3) \rho \varepsilon_{0} \mu_{p}$; then we get

$$
\varepsilon(\omega, T) \approx \frac{\varepsilon_{0}}{1-a \gamma /(-i \omega+\gamma)} .
$$

The real and imaginary parts of the dielectric permittivity are

$$
\begin{gathered}
\varepsilon^{\prime}(\omega, T)=\frac{\varepsilon_{0}\left[(1-a) \gamma^{2}+\omega^{2}\right]}{(1-a)^{2} \gamma^{2}+\omega^{2}}, \\
\varepsilon^{\prime \prime}(\omega, T)=\frac{\varepsilon_{0} a \gamma \omega}{(1-a)^{2} \gamma^{2}+\omega^{2}} .
\end{gathered}
$$

The measured temperature dependent $\varepsilon^{\prime}(T)$ and $\varepsilon^{\prime \prime}(T)$ (see Figures 7, 8, and 9) for CCTO, BFN, and CFN are similar to those reported in previous work $[3-7,30]$. The real part dielectric permittivity $\varepsilon^{\prime}$ reveals a pronounced steplike increase accompanied by a loss peak. The dielectric permittivity of CCTO, BFN, and CFN is fitted according to (7) by a least-square method. For CCTO measured at $100 \mathrm{kHz}$, the best fitted result was obtained with $a=0.971$, $\gamma_{0}=3.4 \times 10^{9}$, and $\Delta=924 \mathrm{~K}$. It is very interesting to note that the obtained barrier energy $E$ (obtained from $\Delta=$ $\left.E / k_{B}\right)$ is $0.08 \mathrm{eV}$, a value quite close to the characteristic 

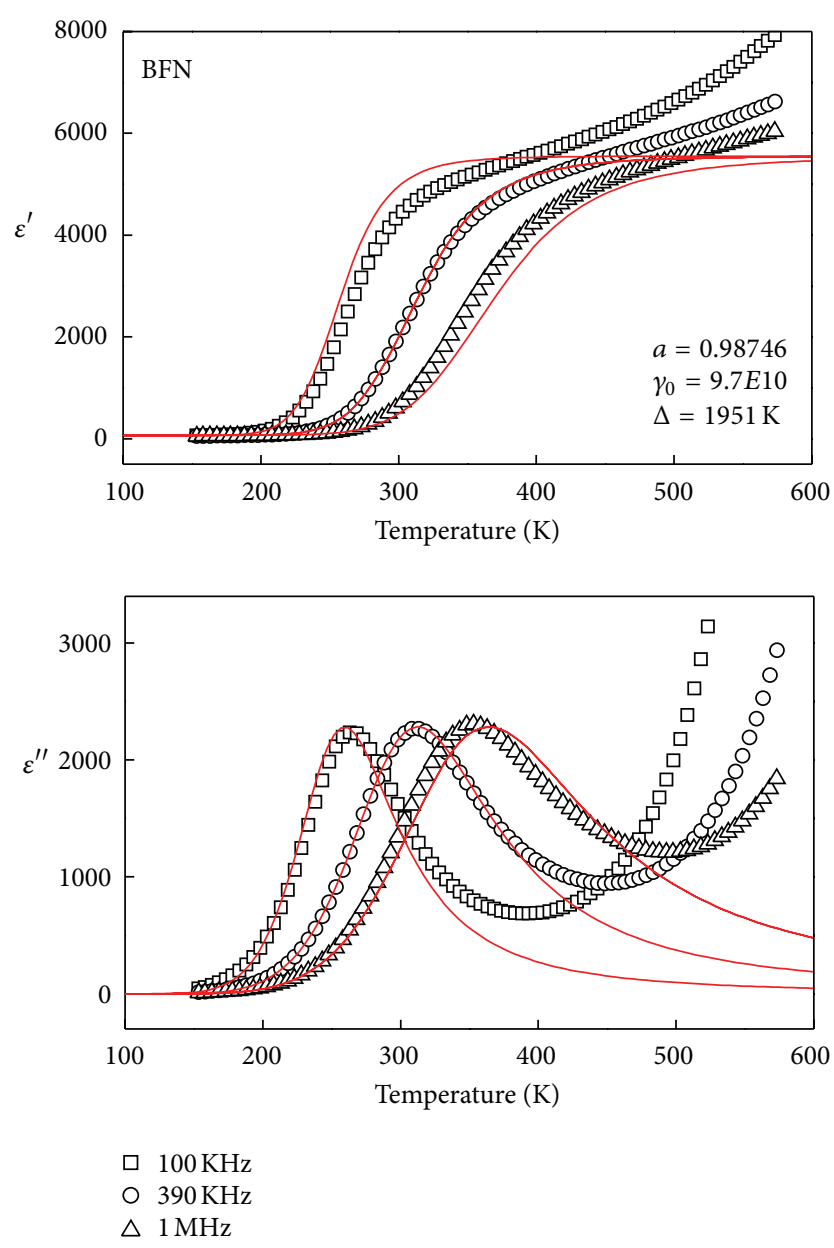

FIGURE 8: The dielectric constant of BFN is shown against temperature. The solid lines are the beat fits of polaron model.

activation energy obtained from (2) (Section 3.2, $E_{a}=$ $0.1 \mathrm{eV}$ for CCTO). The same fitting parameters were used to calculate the temperature dependence of $\varepsilon^{\prime}$ and $\varepsilon^{\prime \prime}$ at different frequencies. The solid lines in Figure 7 are calculated from the previous model, which reproduce the experimental data quite well. We have also performed the least square fitting process on BFN and CFN. The best result was obtained with $a=0.987, \gamma_{0}=9.7 E 10$, and $\Delta=1951 \mathrm{~K}$ for $\mathrm{BFN}$, and $a=0.987, \gamma_{0}=1.98 E 9$, and $\Delta=2695 \mathrm{~K}$ for $\mathrm{CFN}$, respectively. The calculated barrier energy $E$ is $0.17 \mathrm{eV}$ for $\mathrm{BFN}$, which is also quite close to the result obtained from (2) (see Figure 5). However, the calculated barrier energy for CFN is only $0.24 \mathrm{eV}$, which is much smaller than the one obtained from (2) $(0.34 \mathrm{eV})$. Presumably, this may be attributed to an overlap between the polaron relaxation and a high temperature relaxation process as shown in Figure 9.

\section{Conclusions}

In summary, we have prepared single phase CCTO and AFN (BFN, CFN, and SFN) ceramics by conventional solid state reaction. Based on the dielectric study, it can be concluded

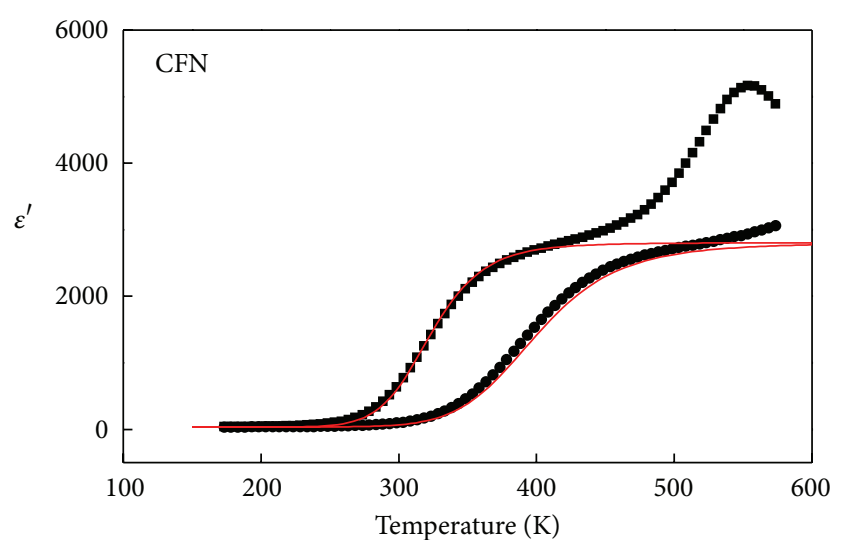

- $1 \mathrm{KHz}$

- $10 \mathrm{KHz}$

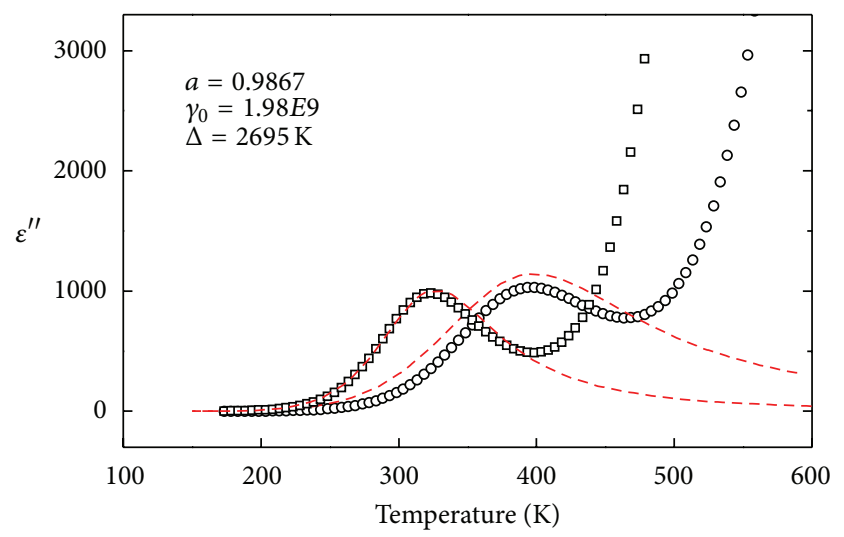

FIgURE 9: The dielectric constant of CFN is shown as a function of temperature. The solid lines are the beat fits of polaron model.

that the low temperature step-like dielectric relaxation in CCTO and AFN can be well explained by a polaron model involving a dipolar-type relaxation. By using a mean-field approach, we get a dielectric function which describes the dielectric relaxation in CCTO and BFN quite well.

\section{Acknowledgments}

This work was supported by the Hong Kong Polytechnic University (Projects nos. A-PL54 and 1-BD08), the National Natural Science Foundations of China (nos. 51302172 and 51272161), and Shenzhen Innovation and Technology Commission under the strategic emerging industries development project (Contract no. ZDSY20120612094418467).

\section{References}

[1] L. Kerkache, A. Layadi, E. Dogheche, and D. Remiens, "Structural, ferroelectric and dielectric properties of $\operatorname{In}_{2} \mathrm{O}_{3}: \mathrm{Sn}$ (ITO) on $\mathrm{PbZr}_{0.53} \mathrm{Ti}_{0.47} \mathrm{O}_{3}$ (PZT)/Pt and annealing effect," Journal of Alloys and Compounds, vol. 509, no. 20, pp. 6072-6076, 2011.

[2] N. Vittayakorn, G. Rujijanagul, and D. P. Cann, "Investigation of the influence of thermal treatment on the morphologies, dielectric and ferroelectric properties of PZT-based ceramics," 
Journal of Alloys and Compounds, vol. 440, no. 1-2, pp. 259-264, 2007.

[3] M. A. Subramanian, D. Li, N. Duan, B. A. Reisner, and A. W. Sleight, "High dielectric constant in $\mathrm{ACu}_{3} \mathrm{Ti}_{4} \mathrm{O}_{12}$ and $\mathrm{ACu}_{3} \mathrm{Ti}_{3} \mathrm{FeO}_{12}$ phases," Journal of Solid State Chemistry, vol. 151, no. 2, pp. 323-325, 2000.

[4] D. C. Sinclair, T. B. Adams, F. D. Morrison, and A. R. West, " $\mathrm{CaCu}_{3} \mathrm{Ti}_{4} \mathrm{O}_{12}$ : one-step internal barrier layer capacitor," Applied Physics Letters, vol. 80, no. 12, pp. 2153-2155, 2002.

[5] A. P. Ramirez, M. A. Subramanian, M. Gardel et al., "Giant dielectric constant response in a copper-titanate," Solid State Communications, vol. 115, no. 5, pp. 217-220, 2000.

[6] P. Lunkenheimer, R. Fichtl, S. G. Ebbinghaus, and A. Loidl, "Nonintrinsic origin of the colossal dielectric constants in $\mathrm{CaCu}_{3} \mathrm{Ti}_{4} \mathrm{O}_{12}$," Physical Review B, vol. 70, no. 17, Article ID 172102, 4 pages, 2004.

[7] C. C. Homes, T. Vogt, S. M. Shapiro, S. Wakimoto, and A. P. Ramirez, "Optical response of high-dielectric-constant perovskite-related oxide," Science, vol. 293, no. 5530, pp. 673676, 2001.

[8] I. P. Raevski, S. A. Prosandeev, A. S. Bogatin, M. A. Malitskaya, and L. Jastrabik, "High dielectric permittivity in $\mathrm{AFe}_{1 / 2} \mathrm{~B}_{1 / 2} \mathrm{O}_{3}$ nonferroelectric perovskite ceramics $(\mathrm{A}=\mathrm{Ba}, \mathrm{Sr}, \mathrm{Ca} ; \mathrm{B}=\mathrm{Nb}$, Ta, Sb)," Journal of Applied Physics, vol. 93, no. 7, pp. 4130-4136, 2003.

[9] K. P. Neupane, J. L. Cohn, H. Terashita, and J. J. Neumeier, "Doping dependence of polaron hopping energies in $\mathrm{La}_{1-x} \mathrm{Ca}_{x} \mathrm{MnO}_{3}(0 \leq x \leq 0.15)$," Physical Review B, vol. 74, no. 14, Article ID 144428, 2006.

[10] R. S. Freitas, J. F. Mitchell, and P. Schiffer, "Magnetodielectric consequences of phase separation in the colossal magnetoresistance manganite $\mathrm{Pr}_{0.7} \mathrm{Ca}_{0.3} \mathrm{MnO}_{3}$, Physical Review B, vol. 72, no. 14, Article ID 144429, 2005.

[11] T. Park, Z. Nussinov, K. R. A. Hazzard et al., "Novel dielectric anomaly in the hole-doped $\mathrm{La}_{2} \mathrm{Cu}_{1-x} \mathrm{Li}_{x} \mathrm{O}_{4}$ and $\mathrm{La}_{2-x} \mathrm{Sr}_{x} \mathrm{NiO}_{4}$ insulators: signature of an electronic glassy state," Physical Review Letters, vol. 94, no. 1, Article ID 017002, 2005.

[12] J. Rivas, B. Rivas-murias, A. Fondado, J. Mira, and M. A. Señarís-Rodríguez, "Dielectric response of the charge-ordered two-dimensional nickelate $\mathrm{La}_{1.5} \mathrm{Sr}_{0.5} \mathrm{NiO}_{4}$," Applied Physics Letters, vol. 85, no. 25, Article ID 6224, 3 pages, 2004.

[13] C. C. Wang, Y. M. Cui, and L. W. Zhang, "Dielectric properties of $\mathrm{TbMnO}_{3}$ ceramics," Applied Physics Letters, vol. 90, no. 1, Article ID 012904, 3 pages, 2007.

[14] J. B. Wu, C. W. Nan, Y. H. Lin, and Y. Deng, "Giant dielectric permittivity observed in Li and Ti doped NiO," Physical Review Letters, vol. 89, no. 21, Article ID 217601, 2002.

[15] J. L. Cohn, M. Peterca, and J. J. Neumeier, "Low-temperature permittivity of insulating perovskite manganites," Physical Review B, vol. 70, no. 21, Article ID 214433, pp. 1-6, 2004.

[16] A. Seeger, P. Lunkenheimer, J. Hemberger et al., "Charge carrier localization in $\mathrm{La}_{1-x} \mathrm{Sr}_{x} \mathrm{MnO}_{3}$ investigated by ac conductivity measurements," Journal of Physics Condensed Matter, vol. 11, no. 16, pp. 3273-3290, 1999.

[17] J. L. Cohn, M. Peterca, and J. J. Neumeier, "Giant dielectric permittivity of electron-doped manganite thin films, $\mathrm{Ca}_{1-x} \mathrm{LaxO}_{3}$ $(0 \leq x \leq 0.03)$," Journal of Applied Physics, vol. 97, no. 3, Article ID 034102, 2005.

[18] L. Zhang and Z. J. Tang, "Polaron relaxation and variable-rangehopping conductivity in the giant-dielectric-constant material $\mathrm{CaCu}_{3} \mathrm{Ti}_{4} \mathrm{O}_{12}$," Physical Review B, vol. 70, no. 17, Article ID 174306, 6 pages, 2004.
[19] A. Tselév, C. M. Brooks, S. M. Anlage et al., "Evidence for powerlaw frequency dependence of intrinsic dielectric response in the $\mathrm{CaCu}_{3} \mathrm{Ti}_{4} \mathrm{O}_{12}$," Physical Review B, vol. 70, no. 14, Article ID 144101, 2004.

[20] C. C. Wang and L. W. Zhang, "Polaron relaxation related to localized charge carriers in $\mathrm{CaCu}_{3} \mathrm{Ti}_{4} \mathrm{O}_{12}$ " Applied Physics Letters, vol. 90, no. 14, Article ID 142905, 3 pages, 2007.

[21] L. Liu, C. C. Wang, X. H. Sun, G. J. Wang, C. M. Lei, and T. $\mathrm{Li}$, "Oxygen-vacancy-related relaxations of $\mathrm{Sr}_{3} \mathrm{CuNb}_{2} \mathrm{O}_{9}$ at high temperatures," Journal of Alloys and Compounds, vol. 552, pp. 279-282, 2013.

[22] L. Fang, M. R. Shen, F. Zheng, Z. Li, and J. Yang, "Dielectric responses and multirelaxation behaviors of pure and doped $\mathrm{CaCu}_{3} \mathrm{Ti}_{4} \mathrm{O}_{12}$ ceramics," Journal of Applied Physics 104, vol. 104, no. 6, Article ID 064110, 8 pages, 2008.

[23] S. M. Ke, H. T. Huang, and H. Q. Fan, "Relaxor behavior in $\mathrm{CaCu}_{3} \mathrm{Ti}_{4} \mathrm{O}_{12}$ ceramics," Applied Physics Letters, vol. 89, no. 18, Article ID 182904, 3 pages, 2006.

[24] S. Ke, H. Huang, H. Fan, H. L. W. Chan, and L. M. Zhou, "Colossal dielectric response in barium iron niobate ceramics obtained by different precursors," Ceramics International, vol. 34, no. 4, pp. 1059-1062, 2008.

[25] N. Kolev, C. L. Chen, M. Gospodinov et al., "Raman spectroscopy of $\mathrm{CaRuO}_{3}$," Physical Review B, vol. 66, no. 1, Article ID 014101, pp. 141011-141014, 2002.

[26] S. Saha and T. P. Sinha, "Low-temperature scaling behavior of $\mathrm{BaFe}_{0.5} \mathrm{Nb}_{0.5} \mathrm{O}_{3}$," Physical Review B, vol. 65, no. 13, Article ID 134103, 7 pages, 2002.

[27] K. Tezuka, K. Henmi, Y. Hinatsu, and N. M. Masaki, "Magnetic susceptibilities and Mossbauer spectra of perovskites $\mathrm{A}_{2} \mathrm{FeNbO}_{6}(\mathrm{~A}=\mathrm{Sr}, \mathrm{Ba})$," Journal of Solid State Chemistry, vol. 154, no. 2, pp. 591-597, 2000.

[28] Y. Y. Liu, X. M. Chen, X. Q. Liu, and L. Li, "Dielectric relaxations in $\mathrm{Ca}\left(\mathrm{Fe}_{1 / 2} \mathrm{Nb}_{1 / 2}\right) \mathrm{O}_{3}$ complex perovskite ceramics," Applied Physics Letters, vol. 90, no. 26, Article ID 262904, 3 pages, 2007.

[29] A. K. Jonscher, "The universal dielectric response and its physical significance," IEEE Transactions on Electrical Insulation, vol. 27, no. 2, pp. 407-423, 1992.

[30] Z. Wang, X. M. Chen, L. Ni, and X. Q. Liu, "Dielectric abnormities of complex perovskite $\mathrm{Ba}\left(\mathrm{Fe}_{1 / 2} \mathrm{Nb}_{1 / 2}\right) \mathrm{O}_{3}$ ceramics over broad temperature and frequency range," Applied Physics Letters, vol. 90, no. 2, Article ID 022904, 3 pages, 2007.

[31] P. R. Bueno, M. A. Ramirez, J. A. Varela, and E. Longo, "Dielectric spectroscopy analysis of $\mathrm{CaCu}_{3} \mathrm{Ti}_{4} \mathrm{O}_{12}$ polycrystalline systems," Applied Physics Letters, vol. 89, no. 19, Article ID 191117, 3 pages, 2006.

[32] S. Ke, H. Fan, and H. Huang, "Dielectric relaxation in $\mathrm{A}_{2} \mathrm{FeNbO}_{6}(\mathrm{~A}=\mathrm{Ba}, \mathrm{Sr}$, and $\mathrm{Ca})$ perovskite ceramics," Journal of Electroceramics, vol. 22, no. 1-3, pp. 252-256, 2009.

[33] A. P. Ramirez, G. Lawes, V. Butko, M. A. Subramanian, and C. M. Varma, "Colossal dielectric constants in braced latticeswith defects," http://arxiv.org/abs/cond-mat/0209498 .

[34] B. I. Halperin and C. M. Varma, "Defects and the central peak near structural phase transitions," Physical Review B, vol. 14, no. 9, pp. 4030-4044, 1976. 

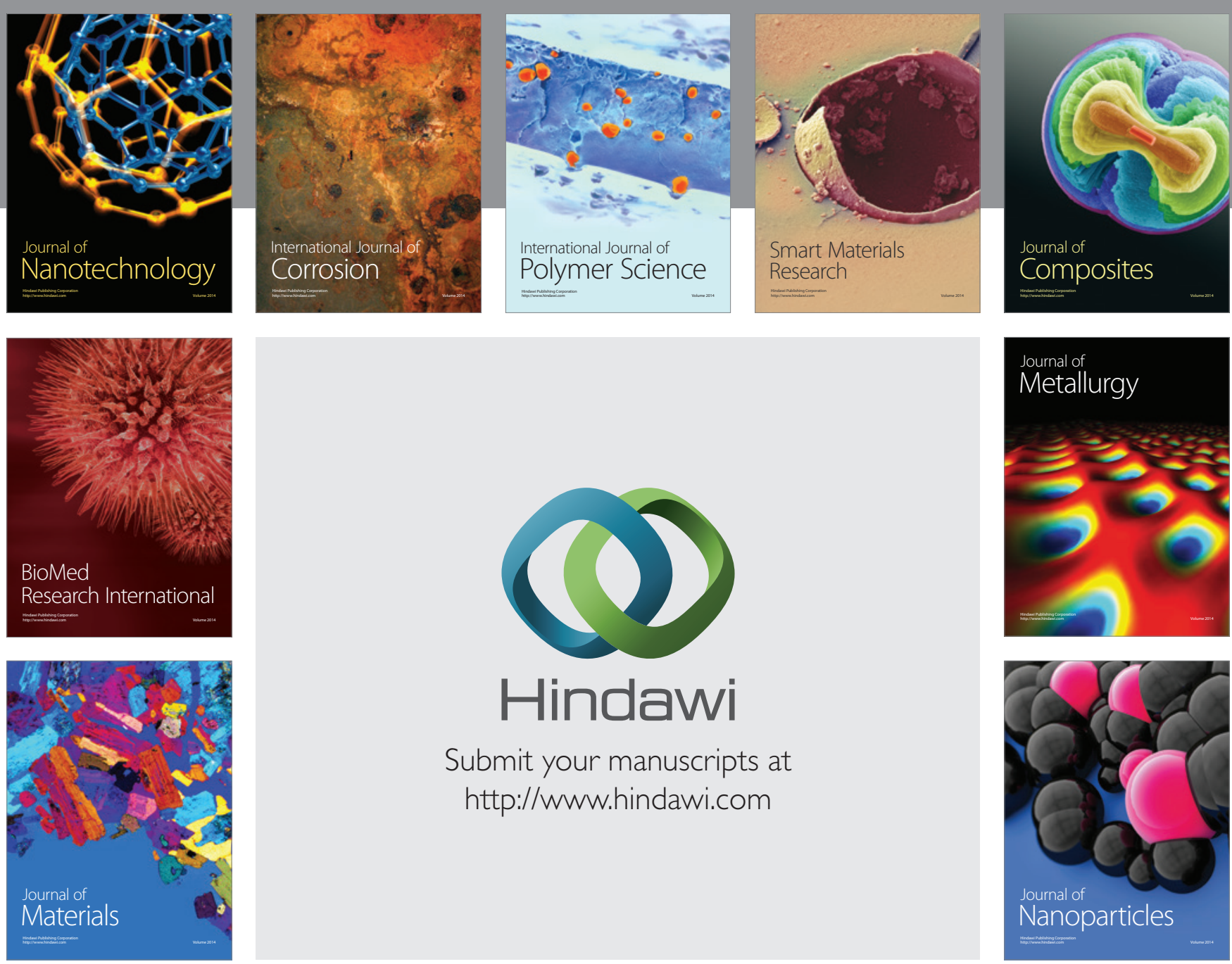

Submit your manuscripts at http://www.hindawi.com
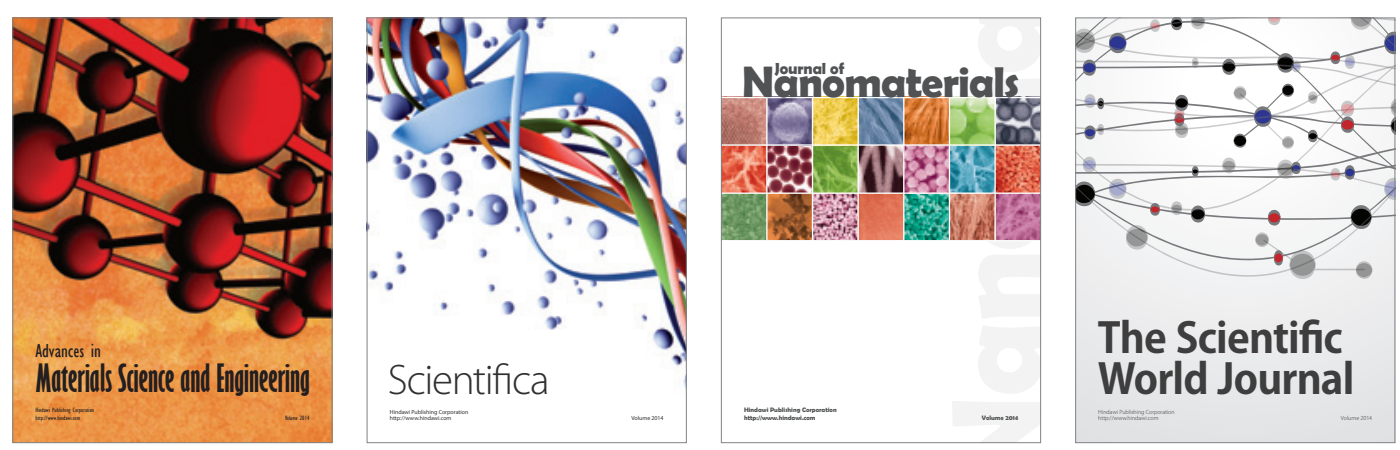

\section{The Scientific World Journal}
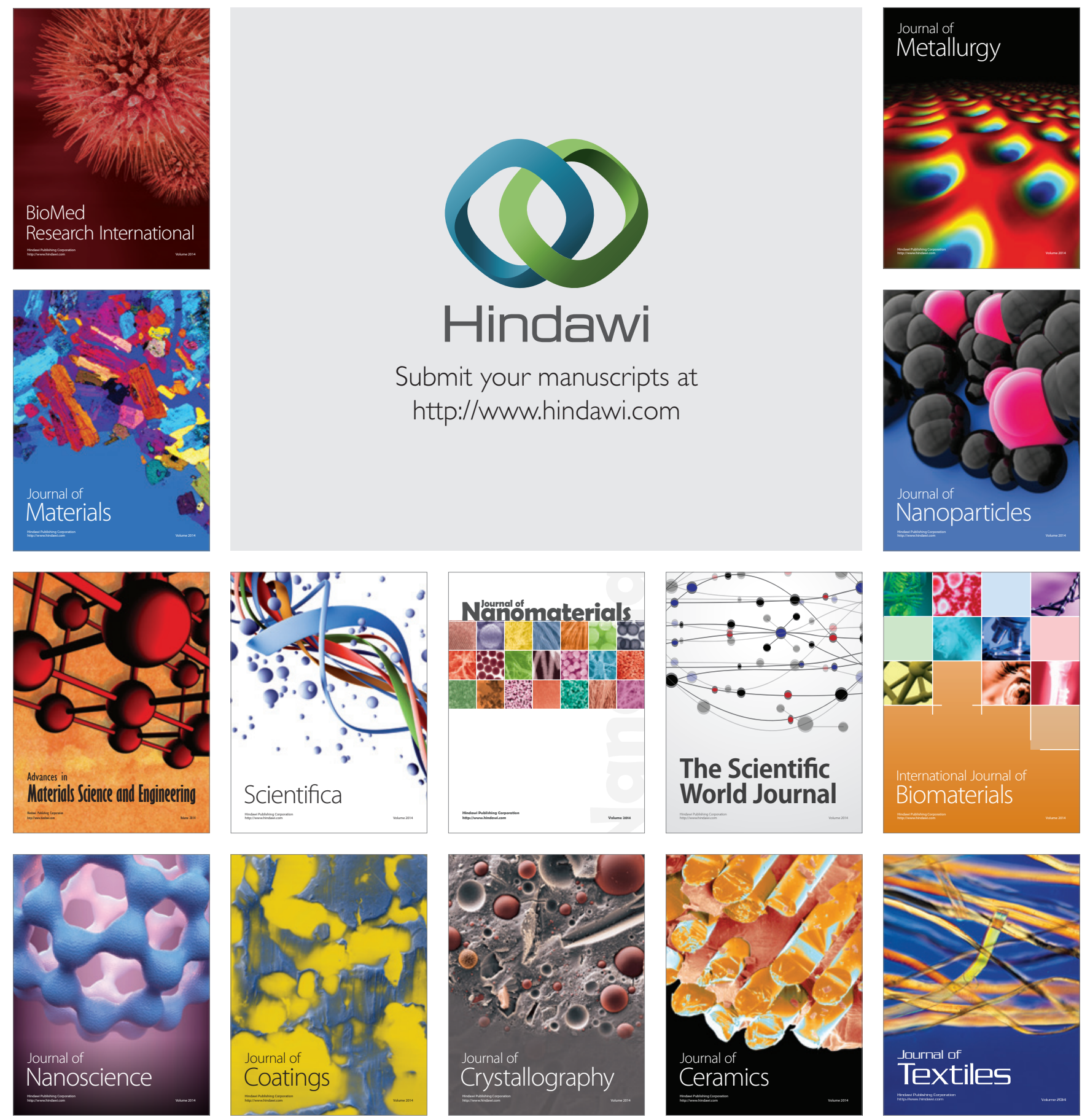